$517.949: 534.121 .3$

\title{
A Numerical Calculation by Finite Difference Method for Free Vibration Problem of Axisymmetric Shells*
}

\author{
By Minoru HamadA** and Katsuo Miyata***
}

\begin{abstract}
B. Budiansky and P.P. Radkowski(1) once presented a finite difference method for problems of unsymmetrical bending of axisymmetric shells. By putting the terms of inertia into the equilibrium equations in their paper, a numerical method for free vibration problems of axisymmetric shells of arbitrary forms is proposed in this study, and the validity of this method is checked for shells of various kinds-circular plates, cylindrical shells, conical shells, spherical shells, and shells of nuclear vessels.
\end{abstract}

\section{Introduction}

The theory presented by B. Budiansky and P.P. Radkowski(1) for statical bending problems of axisymmetric shells is based on the general linear shell theory of J.L. Jr. Sanders ${ }^{(2)}$. Their theory utilizes the method of numerical calculation by finite difference, and makes it possible to calculate stress distributions and deformations of axisymmetric shells of arbitrary forms. In this paper, a numerical method for a free vibration problem of any axisymmetric shell is obtained by putting the terms of inertia into the equilibrium equations in their theory. In this problem, the value of a determinant of very.high degree should be estimated. For this purpose, the elimination process is applied, and the validity of this method is checked for shells of various kinds.

\section{Analytical formulations}

The following notations are used without interpretation.

$a=$ reference length

$h=$ thickness which is assumed as partially constant

$h_{0}=$ reference thickness

$n=$ Fourier index

$r=$ normal distance from shell to axis (Fig. 1)

$s=$ meridional shell coordinate (Fig. 1)

$t=$ time

* Received 6th January, 1971.

** Professor, Faculty of Engineering, Osaka University, Suita.

*** Engineer, Sumitomo Light Metal Industries, Ltd.
$E=$ Young's modulus

$M_{\xi}, M_{\theta}, M_{\xi \theta}=$ bending moment per unit length (Fig. 2)

$\bar{M}_{\xi \theta}=$ modified twisting moment

$N_{\xi}, N_{\theta}, N_{\xi \theta}=$ membrane forces per unit length (Fig. 2)

$\bar{N}_{\xi \theta}=$ modified membrane shear

$\hat{N}_{\xi \theta}=$ effective (boundary) membrane shear (Fig. 4)

$Q_{\xi}, Q_{\theta}=$ transverse forces per unit length (Fig. 2)

$\hat{Q}_{\xi}=$ effective (boundary) transverse shear (Fig. 4)

$R_{\xi}, R_{\theta}=$ radii of curvature (Fig. 1)

$W, U_{\xi}, U_{\theta}=$ normal, meridional and circumferential displacements (Fig. 3 )

$$
\gamma=\rho^{\prime} / \rho
$$

$\varepsilon_{\xi}, \dot{\varepsilon}_{\theta}, \varepsilon_{\xi \theta}=$ membrane strains

$\theta=$ circumferential angle (Fig. 1)

$\kappa_{\xi}, \kappa_{\theta}, \kappa_{\xi \theta}=$ bending distortions

$\lambda=h_{0} / a$

$\nu=$ Poisson's ratio

$\xi=$ nondimensional meridional coordinates $(s / a)$

$\rho=r / a$

$\rho^{*}=$ mass density

$\sigma_{\xi}, \sigma_{\theta}, \sigma_{\xi \theta}=$ meridional, circumferential and shear stresses
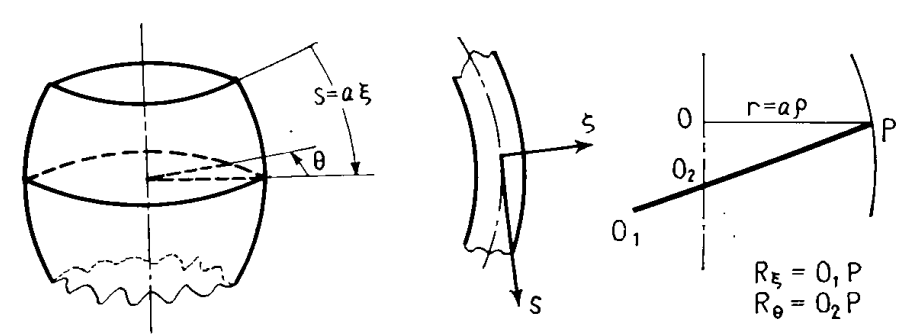

Fig. 1 Surface geometry and coordinates 
$\sigma_{0}=$ reference stress

$\tau=$ nondimensional time $=(t / a) \sqrt{E} / \rho^{*}$

$\omega_{\theta}, \omega_{\xi}=$ nondimensional curvatures $\left(=a / R_{\theta}, a / R_{\xi}\right)$

$\zeta=$ normal coordinate from shell middle surface (Fig. 1)

$\Phi_{\xi}, \Phi_{\theta}=$ rotations (Fig. 3)

$'($ prime $)=d / d \xi$

For an axisymmetric shell, the following geometric relations are satisfied:

$\left.\omega_{\theta}=\sqrt{1-(} \rho^{\prime}\right)^{2} / \rho, \quad \omega_{\xi}=-\left(\gamma^{\prime}+\gamma^{2}\right) / \omega_{\theta}, \quad \omega_{\theta}^{\prime}=\gamma\left(\omega_{\xi}-\omega_{\theta}\right), \quad \rho^{\prime \prime} / \rho=-\omega_{\xi} \omega_{\theta}$

In the shell theory of Sanders ${ }^{(2)}$, the modified twisting moment $\bar{M}_{\xi \theta}$ and the modified membrane shear $\bar{N}_{\xi \theta}$ are used, which are defined by

$$
\left.\begin{array}{l}
\bar{M}_{\xi \theta}=\left(M_{\xi \theta}+M_{\theta \xi}\right) / 2 \\
\bar{N}_{\xi \theta}=\left(N_{\xi \theta}+N_{\theta \xi}\right) / 2+\left\{\left(1 / R_{\theta}\right)-\left(1 / R_{\xi}\right)\right\}\left(M_{\xi \theta}-M_{\theta \xi}\right) / 4
\end{array}\right\}
$$

Putting the terms of inertia into the equilibrium equations in the Sanders theory and eliminating transvers forces $Q_{\xi}$ and $Q_{\theta}$, we obtain the following equations:

$$
\begin{gathered}
a\left[\frac{\partial}{\partial \xi}\left(\rho_{\xi}\right)+\frac{\partial}{\partial \theta}\left(\bar{N}_{\xi \theta}\right)-\rho^{\prime} N_{\theta}\right]+\omega_{\xi}\left[\frac{\partial}{\partial \xi}\left(\rho M_{\xi}\right)+\frac{\partial}{\partial \theta}\left(\bar{M}_{\xi \theta}\right)\right. \\
\left.\quad-\rho^{\prime} M_{\theta}\right]+\frac{1}{2}\left(\omega_{\xi}-\omega_{\theta}\right) \frac{\partial}{\partial \theta}\left(\bar{M}_{\xi \theta}\right)-E \rho h \frac{\partial^{2} U_{\xi}}{\partial \tau^{2}}=0 \\
a\left[\frac{\partial}{\partial \theta}\left(N_{\theta}\right)+\frac{\partial}{\partial \xi}\left(\rho \bar{N}_{\xi \theta}\right)+\rho^{\prime} \bar{N}_{\xi \theta}\right]+\omega_{\theta}\left[\frac{\partial}{\partial \theta}\left(M_{\theta}\right)+\frac{\partial}{\partial \xi}\left(\rho \bar{M}_{\xi \theta}\right)\right. \\
\left.\quad+\rho^{\prime} \bar{M}_{\xi \theta}\right]+\frac{\rho}{2} \frac{\partial}{\partial \xi}\left[\left(\omega_{\theta}-\omega_{\xi}\right) \bar{M}_{\xi \theta}\right]-E \rho h \frac{\partial^{2} U_{\theta}}{\partial \tau^{2}}=0 \\
\frac{\partial}{\partial \xi}\left[\frac{\partial}{\partial \xi}\left(\rho M_{\xi}\right)+\frac{\partial}{\partial \theta}\left(\bar{M}_{\xi \theta}\right)-\rho^{\prime} M_{\theta}\right]+\frac{1}{\rho}-\frac{\partial}{\partial \theta}\left[\frac{\partial}{\partial \theta}\left(M_{\theta}\right)+\frac{\partial}{\partial \xi}\left(\rho \bar{M}_{\xi \theta}\right)\right. \\
\left.\quad+\rho^{\prime} \bar{M}_{\xi \theta}\right]-a \rho\left(\omega_{\xi} N_{\xi}+\omega_{\theta} N_{\theta}\right)-E \rho h \frac{\partial^{2} W}{\partial \tau^{2}}=0
\end{gathered}
$$

where the terms of rotatory inertia are neglected. There are another 14 fundamental equations i.e. two relations between $\left(\Phi_{\xi}, \Phi_{\theta}\right)$ and $\left(W, U_{\xi}, U_{\theta}\right)$, three relations between $\left(\varepsilon_{\xi}, \varepsilon_{\theta}, \varepsilon_{\xi \theta}\right)$ and $\left(W, U_{\xi}, U_{\theta}\right)$, three relations between $\left(\kappa_{\xi}, \kappa_{\theta}, \kappa_{\xi \theta}\right)$ and $\left(\Phi_{\xi}, \Phi_{\theta}, U_{\xi}, U_{\theta}\right)$, three relations between ( $\left.\varepsilon_{\xi}, \varepsilon_{\theta}, \varepsilon_{\xi \theta}\right)$ and $\left(N_{\xi}, N_{\theta}, \bar{N}_{\xi \theta}\right)$, and three relations between $\left(\kappa_{\xi}, \kappa_{\theta}, \kappa_{\xi \theta}\right)$ and $\left(M_{\xi}, M_{\theta}, \bar{M}_{\xi \theta}\right)$. For these 17 fundamental equations, there are 17 dependent variables, which are represented in nondimensional forms as follows:

$N_{\xi}=\sigma_{0} h_{0} t_{\xi} \cos n \theta \cos \Omega \tau, \quad N_{\theta}=\sigma_{0} h_{0} t_{\theta} \cos n \theta \cos \Omega \tau$,

$\bar{N}_{\xi \theta}=\sigma_{0} h_{0} t_{\xi \theta} \sin n \theta \cos \Omega \tau, \quad M_{\xi}=\left(\sigma_{0} h_{0}{ }^{3} / a\right) m_{\xi} \cos n \theta \cos \Omega \tau$,

$M_{\theta}=\left(\sigma_{0} h^{3} / a\right) m_{\theta} \cos n \theta \cos \Omega \tau, \quad \bar{M}_{\xi \theta}=\left(\sigma_{0} h_{0}{ }^{3} / a\right) m_{\xi \theta} \sin n \theta \cos \Omega \tau$,

$U_{\xi}=\left(a \sigma_{0} / E\right) u_{\xi} \cos n \theta \cos \Omega \tau, \quad U_{\theta}=\left(a \sigma_{0} / E\right) u_{\theta} \sin n \theta \cos \Omega \tau$,

$W=\left(a \sigma_{0} / E\right) w \cos n \theta \cos \Omega \tau, \quad \Phi_{\xi}=\left(\sigma_{0} / E\right) \varphi_{\xi} \cos n \theta \cos \Omega \tau$

$\Phi_{\theta}=\left(\sigma_{0} / E\right) \varphi_{\theta} \sin n \theta \cos \Omega \tau, \quad \varepsilon_{\xi}=\left(\sigma_{0} / E\right) e_{\xi} \cos n \theta \cos \Omega \tau$

$\varepsilon_{\theta}=\left(\sigma_{0} / E\right) e_{\theta} \cos n \theta \cos \Omega \tau, \quad \varepsilon_{\xi \theta}=\left(\sigma_{0} / E\right) e_{\xi \theta} \sin n \theta \cos \Omega \tau$,

$\kappa_{\xi}=\left(\sigma_{0} / a E\right) k_{\xi} \cos n \theta \cos \Omega \tau, \quad \kappa_{\theta}=\left(\sigma_{0} / a E\right) k_{\theta} \cos n \theta \cos \Omega \tau$,

$\kappa_{\xi \theta}=\left(\sigma_{0} / a E\right) k_{\xi \theta} \sin n \theta \cos \Omega \tau$

where $\Omega$ is a nondimensional circular frequency, and it is related to the natural frequency $f$ by

$$
\Omega=2 \pi a f \sqrt{\rho^{*} / E}
$$

$$
\}
$$

Now, substitution of Eqs. (4) into the 17 fundamental equations leads to 17 equations with the 17 nondimensional dependent variables $t_{\xi} \sim \kappa_{\xi \theta}$, all of which are functions of $\xi$ only. From these equations, the following four differential equations with four dependent variables, $u_{\xi}, u_{\theta}, w$ and $m_{\xi}$ are derived by elimination:

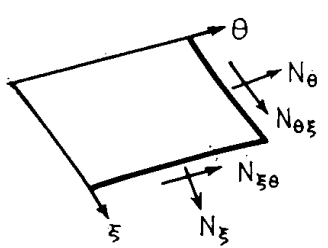

(a)

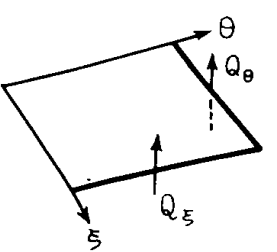

(b)

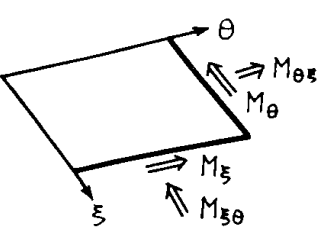

(c)

Fig. 2 Forces and moments

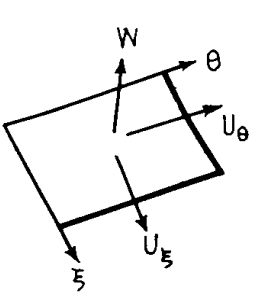

(a)

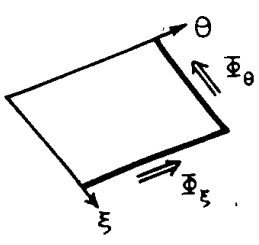

(b)

Fig. 3 Displacements and rotations 


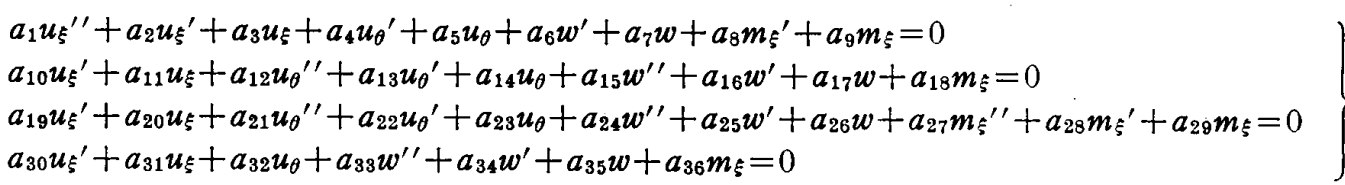

where the coefficients $a_{1} \sim a_{36}$ are determined from the form and the material of the shell, and the natural frequency. Three $a$ 's, which are different from those in the paper of Budiansky and Radkowski ${ }^{(1)}$, are shown as

$$
\begin{aligned}
& a_{3}=-\nu b \omega_{\xi} \omega_{\theta}-b \gamma^{2}-\frac{(1-\nu) b n^{2}}{2 \rho^{2}}+\lambda^{2} d(1-\nu)\left[(1+\nu) \gamma^{2} \omega_{\xi}^{2}+\frac{\left(3 \omega_{\xi}-\omega_{\theta}\right)^{2} n^{2}}{8 \rho^{2}}\right]+e \Omega^{2} \\
& a_{14}=-\gamma a_{13}+\frac{1-\nu}{2} b \omega_{\xi} \omega_{\theta}-\frac{b n^{2}}{\rho^{2}}-\lambda^{2} d(1-\nu)\left[\frac{(1+\nu) \omega_{\theta}^{2} n^{2}}{\rho^{2}}-\frac{\omega_{\xi} \omega_{\theta}}{8}\left(3 \omega_{\theta}-\omega_{\xi}\right)^{2}\right]+e \Omega^{2} \\
& \left.a_{26}=-b\left(\omega_{\xi}^{2}+2 \nu \omega_{\xi} \omega_{\theta}+\omega_{\theta}^{2}\right)+\frac{\lambda^{2} d(1-\nu) n^{2}}{\rho^{2}}\left[(1+\nu)\left(\omega_{\xi} \omega_{\theta}-\frac{n^{2}}{\rho^{2}}+2 \gamma^{2}\right)+2\left(\gamma^{2}+\omega_{\xi} \omega_{\theta}\right)\right]+e \Omega^{2}\right]
\end{aligned}
$$

where

$$
\left.\begin{array}{l}
\lambda=h_{0} / a, e=h / h_{0}, \\
b=e /\left(1-\nu^{2}\right), d=e^{3} / 12\left(1-\nu^{2}\right)
\end{array}\right\}
$$

The other $33 a$ 's are the same as those in ref. (1).

In the Sanders theory, the expression for virtual work per unit length at the boundaries $s=0, \bar{s}$ is

$$
\pm\left(N_{\xi} U_{\xi}+\hat{N}_{\xi \theta} U_{\theta}+\hat{Q}_{\xi} W+M_{\xi} \Phi_{\xi}\right)
$$

where

$$
\left.\begin{array}{l}
\hat{N}_{\xi \theta}=\bar{N}_{\xi \theta}+\frac{1}{2}\left(\frac{3}{R_{\theta}}-\frac{1}{R_{\xi}}\right) \bar{M}_{\xi \theta} \\
\hat{Q}_{\xi}=\frac{1}{a \rho}\left[\frac{\partial}{\partial \xi}\left(\rho M_{\xi}\right)+2 \frac{\partial}{\partial \theta}\left(\bar{M}_{\xi \theta}\right)-\rho^{\prime} M_{\theta}\right]
\end{array}\right\}
$$

(see Fig. 4). $\hat{N}_{\xi \theta}$ and $\hat{Q}_{\xi \theta}$ are written in nondimensional forms as

$$
\left.\begin{array}{l}
\hat{N}_{\xi \theta}=\sigma_{0} h_{0} \hat{t}_{\xi \theta} \sin n \theta \cos \Omega \tau \\
\hat{Q}_{\xi}=\sigma_{0} h_{0} \hat{f}_{\xi} \cos n \theta \cos \Omega \tau
\end{array}\right\}
$$

Here, $t_{\xi}, \hat{t}_{\xi \theta}$ and $\hat{f}_{\xi}$ are related to $u_{\xi}, u_{\theta}, w$ and $m_{\xi}$ as

$$
\begin{aligned}
& t_{\xi}=b_{1} u_{\xi}^{\prime}+b_{2} u_{\xi}+b_{3} u_{\theta}+b_{4} w \\
& \hat{t}_{\xi \theta}=b_{5} u_{\xi}+b_{6} u_{\theta}+b_{7} u_{\theta}+b_{8} w^{\prime}+b_{9} w \\
& \hat{f}_{\xi}=b_{10} u_{\xi}+b_{11} u_{\theta}+b_{12} u_{\theta} \\
& \quad+b_{13} w^{\prime}+b_{14} w+b_{15} m_{\xi}+b_{16} m_{\xi}
\end{aligned}
$$

where the coefficients $b$ 's are the same as those given in ref. ( 1 ).

Equation ( 9 ) indicates the kinds of boundary conditions. In the problem of free vibration, either $N_{\xi}$ or $U_{\xi}, \hat{N}_{\xi \theta}$ or $U_{\theta}, \hat{Q}_{\xi}$ or $W$ and $M_{\xi}$ or

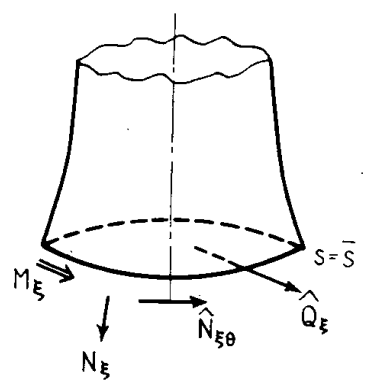

Fig. 4 Effective boundary forces and moments
$\Phi_{\xi}$ vanish. Therefore 16 kinds of boundary conditions are considered. The following four conditions are often used in practical problem:

(1) Free end: $t_{\xi}=\hat{t}_{\xi \theta}=\hat{f}_{\xi}=m_{\xi}=0$

(2) Freely supported end:

$$
t_{\xi}=u_{\theta}=w=m_{\xi}=0
$$

(3) Fixed end:

$$
u_{\xi}=u_{\theta}=w=w^{\prime}=0
$$

(4) Pinned supported end:

$$
u_{\xi}=u_{\theta}=w=m_{\xi}=0
$$

When the shell has a pole, the following conditions should be satisfied at the pole ${ }^{(3)}$ :

$$
\begin{array}{ll}
n=0: & u_{\xi}=u_{\theta}=w^{\prime}=m_{\xi}{ }^{\prime}=0 \\
n=1: & u_{\xi}+u_{\theta}=u_{\theta}{ }^{\prime}=w=m_{\xi}=0 \\
n \geqq 2: & u_{\xi}=u_{\theta}=w=w^{\prime}=0
\end{array}
$$

It can be shown that the conditions $\varphi_{\xi}=0$ and $t_{\xi \theta}=0$ are equivalent to the conditions $w^{\prime}=0$ and $u_{\theta}{ }^{\prime}=0$, respectively.

The differential Eqs. (6) are not valid at points in the shell where discontinuities in geometry occur (Fig. 5). At these points the coefficients $a_{1} \sim a_{36}$ are discontinuous. Ac cordingly, the transition equations are necessary. With II and I superscripts denoting values just beyond and ahead of a discontinuity, respectively, the conditions of geometrical compatibility and equilibrium are

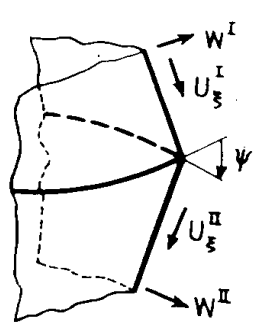

(a)

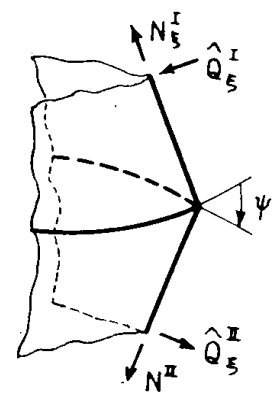

(b)
Fig. 5 Discontinuity conditions 


$$
\begin{aligned}
& u_{\xi}^{\mathrm{I}}=u_{\xi}^{\mathrm{I}} \cos \psi-w^{\mathrm{I}} \sin \phi \\
& u_{\theta}{ }^{\mathrm{I}}=u_{\theta}^{\mathrm{I}} \\
& w^{\mathrm{I}}=u_{\xi}^{\mathrm{I}} \sin \psi+w^{\mathrm{I}} \cos \psi \\
& \varphi_{\xi} \mathrm{II}=\varphi_{\xi} \mathrm{I} \\
& t_{\xi}{ }^{\mathrm{II}}=t_{\xi}^{\mathrm{I}} \cos \psi-\bar{f}_{\xi}^{\mathrm{I}} \sin \psi \\
& \hat{t}_{\xi \theta}{ }^{\mathbb{I}}=\hat{t}_{\xi \theta}{ }^{\mathrm{I}} \\
& \hat{f}_{\xi}^{\mathrm{I}}=t_{\xi}^{\mathrm{I}} \sin \psi+\hat{f}_{\xi}^{\mathrm{I}} \cos \psi \\
& m_{\xi}{ }^{\mathrm{II}}=m_{\xi}^{\mathrm{I}}
\end{aligned}
$$

Also for cases when the shell thickness, the curvature or the material is discontinuous, the conditions in Eqs. (15) and (16) are to be applied. For the branch points as shown in Fig. 6, the transition equations are formulated in the same way as for Eqs. (15) and (16) [see ref. (1)].

Three components of stress are written in nondimensional forms as

$$
\begin{aligned}
& \sigma_{\xi}=\sigma_{0} q_{\xi} \cos n \theta \cos \Omega \tau \\
& \sigma_{\theta}=\sigma_{0} q_{\theta} \cos n \theta \cos \Omega \tau \\
& \sigma_{\xi 0}=\sigma_{\theta} q_{\xi \theta} \sin n \theta \cos \Omega \tau
\end{aligned}
$$

where the nondimensional coefficients $q_{\xi}, q_{\theta}, q_{\xi}$ are expressed by materices as

$$
Q=S \cdot Z^{\prime}+T \cdot Z
$$

where

$$
Q \doteq\left[q_{\xi}, q_{\theta}, q_{\xi \theta}\right]^{T}
$$

and

$$
Z=\left[u_{\xi}, u_{\theta}, w, m_{\xi}\right]^{T}
$$

$S$ and $T$ are $3 \times 4$ matrices, the elements of which are referred to ref. ( 1 ).

\section{Numerical analysis}

Suppose that $p$ discontinuity locations $s=s_{1}$, $s=s_{2} \cdots s=s_{p}$ occur in the range $(0, \bar{s})$ of the shell. Let the regions $\left(0, s_{1}\right),\left(s_{1}, s_{2}\right) \cdots\left(s_{p}, \bar{s}\right)$ be subdivided into $N_{1}-1, N_{2}-1 \cdots N_{p+1}-1$ equal segments, respectively, and the end points of the segments (i.e. the difference points) be identified by the index $j$, running from $j=0$ at $s=0$ to $j=N_{1}$ $+N_{2}+\cdots N_{p_{+1}} \equiv N$ at $s=\bar{s}$, since the discontinuity

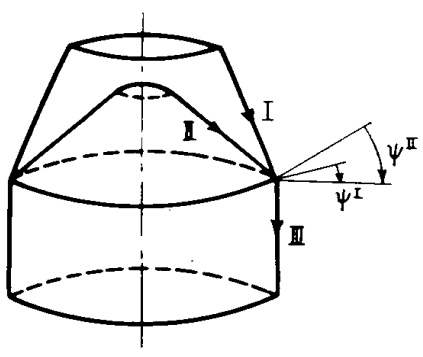

Fig. 6 Branched shell

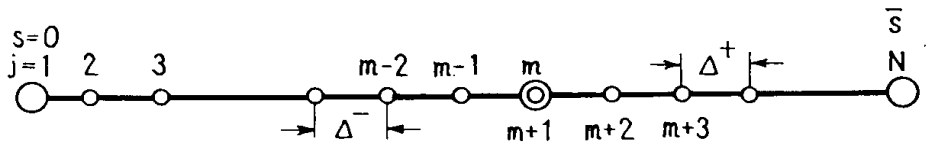

Fig. 7 Numbers of difference points points have double indices in such a way that $j=m$ for one region and $j=m+1$ for another region (Fig. 7). The increments in the non dimensional variable $\xi$ are then

$$
\begin{aligned}
& \Delta_{1}=s_{1} / a\left(N_{1}-1\right) \\
& \Delta_{2}=\left(s_{2}-s_{1}\right) / a\left(N_{2}-1\right) \\
& \cdots \ldots \ldots \ldots \ldots \ldots \ldots \ldots \ldots \ldots \ldots \ldots \ldots \ldots \ldots \ldots \ldots \ldots \\
& \Delta_{p+1}=\left(\bar{s}-s_{p}\right) / a\left(N_{p+1}-1\right)
\end{aligned}
$$

Now, the following formulas of finite difference are applied to the fundamental differential Eqs. ( 6$)$ :

$$
\left.\begin{array}{l}
Z_{j}{ }^{\prime}=\left(Z_{j+1}-Z_{j-1}\right) / 2 \Delta \\
Z_{j}{ }^{\prime \prime}=\left(Z_{j+1}-2 Z_{j}+Z_{j-1}\right) / \Delta^{2}
\end{array}\right\}
$$

Then the approximate difference equation

$$
A_{j} \cdot Z_{j-1}+B_{j} \cdot Z_{j}+C_{j} \cdot Z_{j+1}=0
$$

is obtained, where $A_{j}, B_{j}$ and $C_{j}$ are $4 \times 4$ matrices, the elements of which are determined from the coefficients $a_{1} \sim a_{36}$ at the difference point $j$, and the increment $\Delta$.

For the end points $j=1, j=N$ and the discontinuity points $j=m(j=m+1)$, the following approximating formulas are applied:

$$
\left.\begin{array}{l}
Z_{1}{ }^{\prime}=\left(-3 Z_{1}+4 Z_{2}-Z_{3}\right) / 2 \Delta_{1} \\
Z_{N}{ }^{\prime}=\left(3 Z_{N}-4 Z_{N-1}+Z_{N-2}\right) / 2 \Delta_{p+1}
\end{array}\right\} .
$$

Substitution of Eqs. (24) into Eqs. (13) or Eqs. (14) leads to

$$
(A 1) \cdot Z_{1}+(B 1) \cdot Z_{2}+(C 1) \cdot Z_{3}=0
$$

$(A N) \cdot Z_{N-2}+(B N) \cdot Z_{N-1}+(C N) \cdot Z_{N}=0 \cdots \cdots(27)$ where $(A 1) \sim(C N)$ are $4 \times 4$ matrices, the elements of which are determined for the kinds of the boundary conditions. For the discontinuity points, substitution of Eqs. (25) into Eqs. (15) and (16) leads to

$$
\begin{aligned}
& (A M)_{m} \cdot Z_{m-2}+(B M)_{m} \cdot Z_{m-1}+(C M)_{m} \cdot Z_{m} \\
& \quad+(D M)_{m+1} \cdot Z_{m+1}+(E M)_{m+1} \cdot Z_{m+2} \\
& \quad+(F M)_{m+1} \cdot Z_{m+3}=0 \\
& (G M)_{m} \cdot Z_{m-2}+(H M)_{m} \cdot Z_{m-1}+(I M)_{m} \cdot Z_{m} \\
& \quad+(J M)_{m+1} \cdot Z_{m+1}+(K M)_{m+1} \cdot Z_{m+2} \\
& \quad+(L M)_{m+1} \cdot Z_{m+3}=0
\end{aligned}
$$

where $(A M)_{m} \sim(L M)_{m+1}$ are $4 \times 4$ matrices, the elements of which are determined from the various values at the discontinuity point $j=m$ $(j=m+1)$. For treatment of the branch point (Fig. 6), refer to ref. (1).

Now, in order to calculate the natural frequency, the value of $n$ (a positive integer) is settled, and an appropriate value of $\Omega$ is assumed. Then, Eqs. (23), Eq. (26) and Eq. (27) (with Eqs. (28) in some cases) constitute a system of simultaneous homogeneous algebraic equations. The determinant of the coefficients of these equations should vanish, but since the 
degree of this determinant is very high, calculation of this determinant is difficult. Therefore the following procedures are applied.

The relation

$Z_{j}=X_{j} \cdot Z_{j+1}$

is settled, where $X_{j}$ is a $4 \times 4$ matrix. $X_{1}$ is obtained by elimination of $Z_{3}$ from Eq. (26) and Eq. (23) for $j=2$. From Eq. (29) and Eq. (23)

$$
\left.\begin{array}{l}
A_{j} \cdot Z_{j-1}+B_{j} \cdot Z_{j}+C_{j} \cdot Z_{j+1}=0 \\
Z_{j-1}=X_{j-1} \cdot Z_{j}
\end{array}\right\}
$$

By elimination of $Z_{j-1}$ from Eqs. (30), $X_{j}$ is expressed by $X_{j-1}$. In this way, the values of $X_{j}$ from $j=1$ to $j=N-1$ are obtained.

For the discontinuity points, the following equations including Eqs. (28) are used:

$$
\begin{aligned}
& Z_{m-2}=X_{m-2} Z_{m-1} \\
& Z_{m-1}=X_{m-1} Z_{m} \\
& (A M)_{m} \cdot Z_{m-2}+(B M)_{m} \cdot Z_{m-1}+(C M)_{m} \\
& \quad \times Z_{m}+(D M)_{m+1} \cdot Z_{m+1}+(E M)_{m+1} \cdot Z_{m+2} \\
& \quad+(F M)_{m+1} \cdot Z_{m+3}=0 \\
& \quad(G M)_{m} \cdot Z_{m-2}+(H M)_{m} \cdot Z_{m-1} \\
& \quad+(I M)_{m} \cdot Z_{m}+(J M)_{m+1} \cdot Z_{m+1} \\
& \quad+(K M)_{m+1} \cdot Z_{m+2}+(L M)_{m+1} \cdot Z_{m+3}=0 \\
& A_{m+2} \cdot Z_{m+1}+B_{m+2} \cdot Z_{m+2}+C_{m+2} \cdot Z_{m+3}=0
\end{aligned}
$$

From Eqs. (31), the values $X_{m}$ and $X_{m+1}$ are calculated from $X_{m-2}$ and $X_{m-1}$. Thus, we obtain finally

$$
\left.\begin{array}{l}
Z_{N-2}=X_{N-2} \cdot Z_{N-1} \\
A_{N-1} \cdot Z_{N-2}+B_{N-1} \cdot Z_{N-1}+C_{N-1} \cdot Z_{N}=0 \\
(A N) \cdot Z_{N-2}+(B N) \cdot Z_{N-1}+(C N) \cdot Z_{N}=0
\end{array}\right\}
$$

from Eq. (29), Eq. (23) and Eq. (27)

Now, all of $Z_{j}$ should not vanish, therefore

$$
\mathrm{DET}=\left|\begin{array}{crc}
I & -X_{N-2} & 0 \\
A_{N-1} & B_{N-1} & C_{N-1} \\
(A N) & (B N) & (C N)
\end{array}\right|=0
$$

where $I$ is a $4 \times 4$ unit matrix, and 0 is a $4 \times 4$ zero matrix. If the assumed value of $Q$ satisfies
Eq. (33), then that value is a correct one, i.e. one of the natural frequencies of the shell for the considered value of $n$. Such a value of $\Omega$ is obtained by the trial and error method.

After a value of frequency is found, the coefficients of Eqs. (32) are calculated for that frequency, and the dependent variables at two difference points, $j=N-1$ and $N-2$ are expressed by any one of non-zero dependent variables at the end point (for example, $m_{\xi}$ is settled as a unit for the clamped end). Then, using Eq. (29), the values of the dependent variables at all difference points are obtained. Distribution of the stresses is calculated by Eq. (18).

\section{Examples}

In order to check the validity of the method proposed above, numerical calculations are carried out for circular plates, cylindrical shells, conical shells, spherical shells, and shells of nuclear vessels. In calculations, it is assumed that the thickness is constant $\left(h=h_{0}\right)$, and the shell is made of steel, i.e.

$\rho^{*}=7.9 \times 10^{-10} \mathrm{~kg} \mathrm{sec} / \mathrm{mm}^{4}$

$E=2.1 \times 10^{4} \mathrm{~kg} / \mathrm{mm}^{2}$

$\nu=0.3$

(a) Circular plate with free edge

The notations in Fig. 8 are used. The radius $R$ is chosen as the reference length $a$, then

$$
a=R, \Delta=1 /(N-1), \xi=\rho=r / R=(j-1) \Delta\}
$$$$
\rho^{\prime}=1, \gamma=1 / \rho, \omega_{\theta}=\omega_{\xi}=0
$$

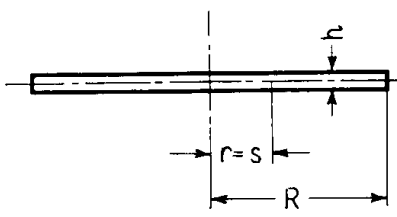

Fig. 8 Circular plate

Table 1 Natural frequencies of circular plates with free edges $(\mathrm{kc} / \mathrm{sec})$

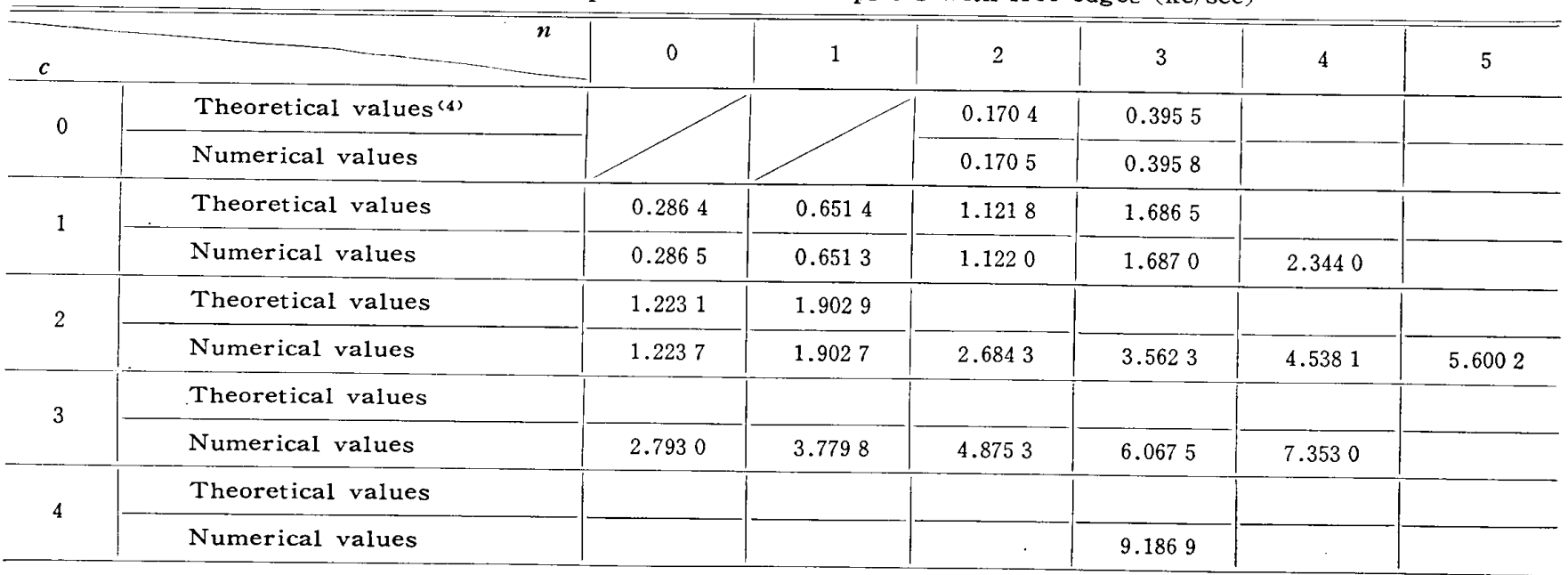


Calculations are carried out for the case of $R=80 \mathrm{~mm}, h=0.82 \mathrm{~mm}$. The results for $N=101$ are shown in Table 1 and Fig. 9, where $c$ is the number of nodal circles. The theoretical values in Table 1 are obtained from ref. (4).

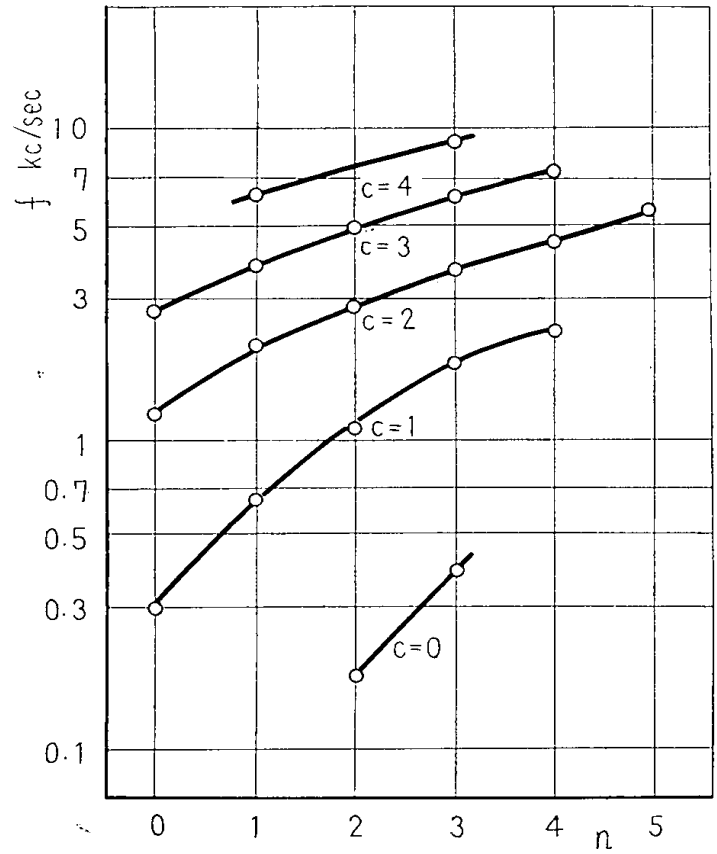

Fig. 9 Natural frequencies of circular plates with free edge $(n=2)$

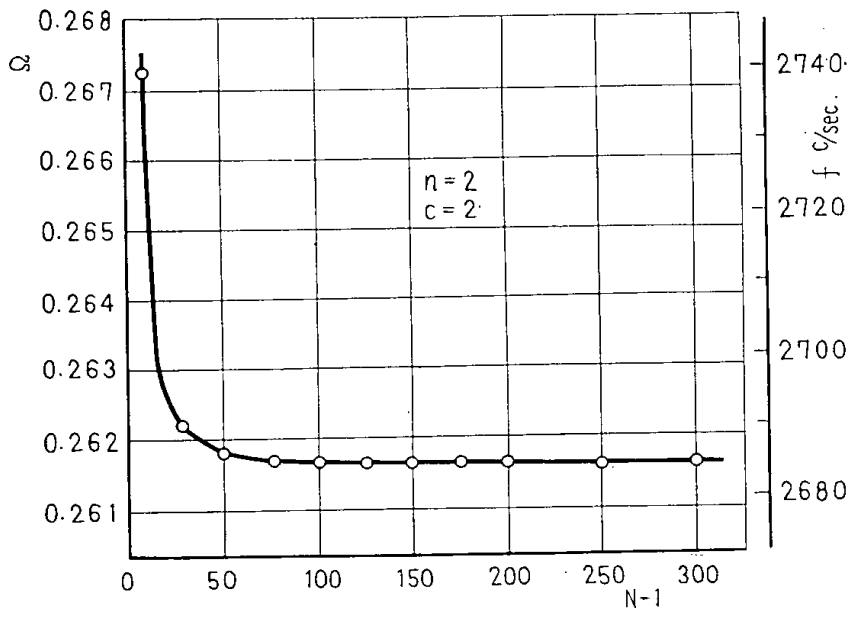

Fig. 10 Effects of $N$ on natural frequency of circular plate

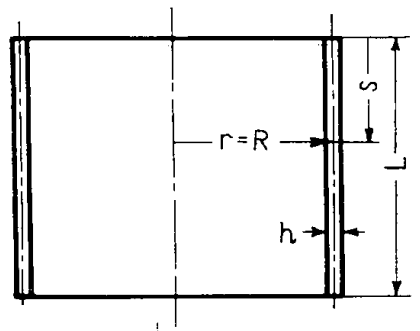

Fig. 11 Cylindrical shell
They coincide well with results obtained by this method. The effect of the number of the segments $N$ on the natural frequency is shown

Table 2 Natural frequencies of cylindrical shells with fixed edges $(n=2)$

\begin{tabular}{c|c|c}
\hline \multirow{2}{*}{$c$} & \multicolumn{2}{|c}{ Numerical values } \\
\cline { 2 - 3 } & $\Omega$ & $f(\mathrm{c} / \mathrm{sec})$ \\
\hline 0 & 0.3277 & 1073.6 \\
\hline 1 & 0.6056 & 1983.7 \\
\hline 2 & 0.7654 & 2507.3 \\
\hline
\end{tabular}

Table 3 Natural frequencies of truncated conical shells with free edges $(n=2)$

\begin{tabular}{|c|c|c|c|c|}
\hline \multirow{2}{*}{$\varphi^{\circ}$} & \multirow{2}{*}{$c$} & \multirow{2}{*}{$\begin{array}{c}\text { Numerical } \\
\text { values } \\
f_{c}(\mathrm{sec} / \mathrm{c})\end{array}$} & \multicolumn{2}{|c|}{ Experimental data } \\
\hline & & & $f_{e}(\mathrm{sec} / \mathrm{c})$ & $h(\mathrm{~mm})$ \\
\hline \multirow{2}{*}{0} & 0 & 66.9 & 67 & \multirow{2}{*}{1.051} \\
\hline & 1 & 86.7 & 91 & \\
\hline \multirow{2}{*}{40} & 0 & 54.1 & & \multirow{2}{*}{0.796} \\
\hline & 1 & 131.1 & 114 & \\
\hline \multirow{2}{*}{80} & 0 & 48.6 & & \\
\hline & 1 & 291.5 & & \\
\hline \multirow{2}{*}{120} & 0 & 51.3 & 60 & \multirow{2}{*}{1.007} \\
\hline & 1 & 862.0 & & \\
\hline \multirow{2}{*}{160} & 0 & 49.5 & & \multirow{2}{*}{1.010} \\
\hline & 1 & 656.5 & 740 & \\
\hline \multirow{2}{*}{180} & 0 & 33.6 & & \multirow{2}{*}{1.000} \\
\hline & 1 & 221.1 & 221 & \\
\hline
\end{tabular}

Table 4 Natural frequencies of partial spherical shells with fixed edge $(n=2)$

\begin{tabular}{c|c|c|c|c}
\hline \multirow{2}{*}{$c$} & \multicolumn{2}{|c|}{ Values by this method } & \multicolumn{2}{|c}{$\begin{array}{c}\text { Values by Kalnins' } \\
\text { method }{ }^{(6)}\end{array}$} \\
\cline { 2 - 4 } & $\Omega$ & $f_{c}(\mathrm{c} / \mathrm{sec})$ & $\Omega$ & $f_{\iota}(\mathrm{c} / \mathrm{sec})$ \\
\hline 0 & 1.4104 & 11573 & 1.472 & 12079 \\
1 & 2.5354 & 20805 & 2.736 & 22451 \\
2 & 4.3426 & 35634 & 4.746 & 38944 \\
3 & 6.1749 & 50670 & 6.442 & 52861 \\
4 & 6.7818 & 55650 & 7.426 & 60936 \\
\hline
\end{tabular}

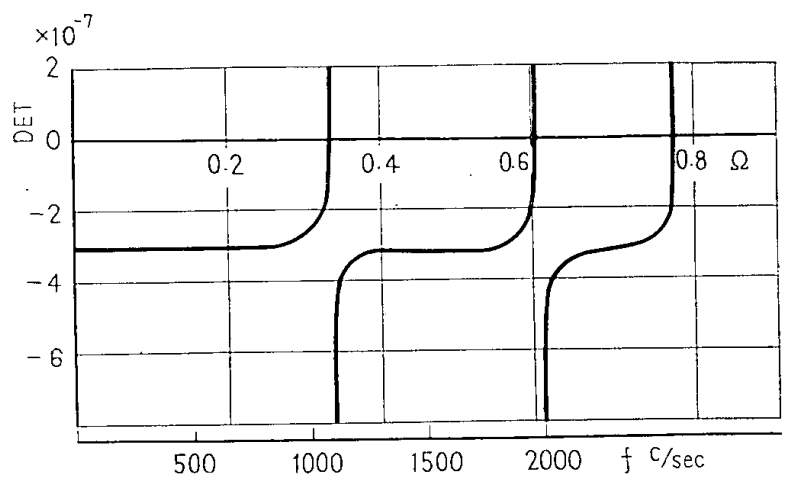

Fig. 12 DET $\Omega$ curves for cylindrical shell with fixed edge $(n=2)$ 
in Fig. 10, which proves that in this case the results for $N \geqq 100$ are accurate enough.

(b) Cylindrical shell with fixed edges

The notations in Fig. 11 are used. The radius $R$ is chosen as the reference length $a$, then

$a=R, \Delta=L / R(N-1), \xi=s / R, \rho=1$,

$\rho^{\prime}=0, \gamma=0, \omega_{\theta}=1, \omega_{\xi}=0$

Calculations are carried out for the case of $R=250.5 \mathrm{~mm}, h=1.0 \mathrm{~mm}, L=600 \mathrm{~mm}$. The results for $n=2, N=101$ are shown in Table 2. A

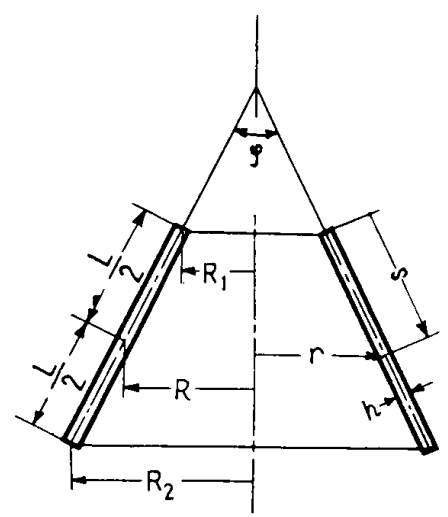

Fig. 13 Truncated conical shell

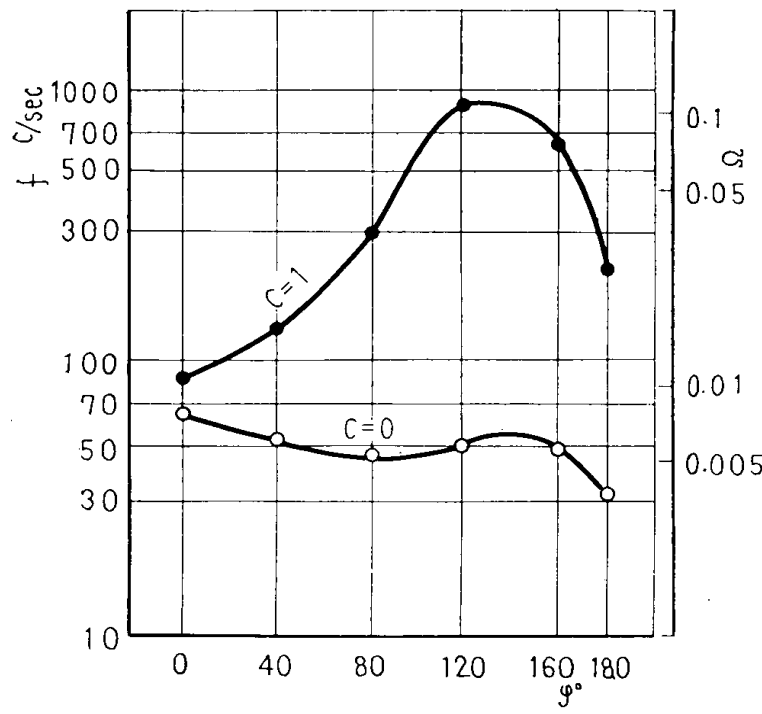

Fig. 14 Natural frequencies of truncated conical shells with free edge $(n=2)$

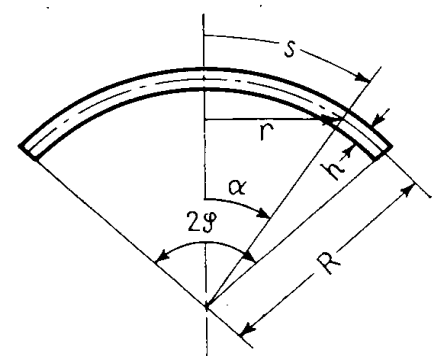

Fig. 15 Partial spherical shell diagram in ref. ( 5 ) gives the result $f=1940$ $(c / s)$ for the case of $c=1$, which coincide well with the corresponding value in Table 2. Figure

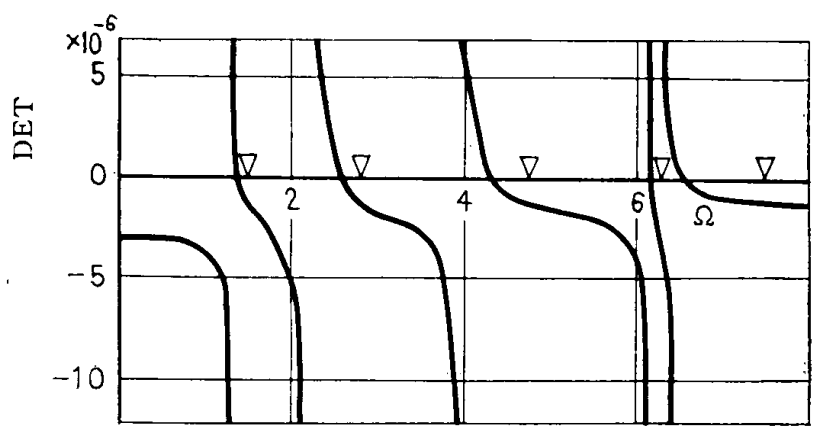

Fig. 16 DET $\sim$ curves for partial spherical shell with fixed edge $(n=2)$ [D: A Kalnin's results $\left.{ }^{(6)}\right]$

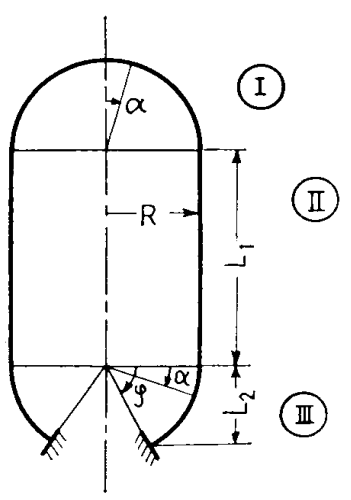

(a)

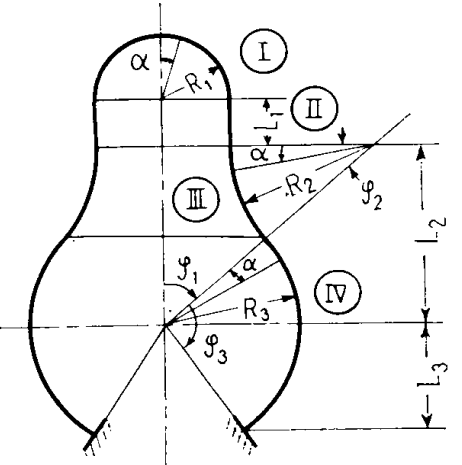

(b)

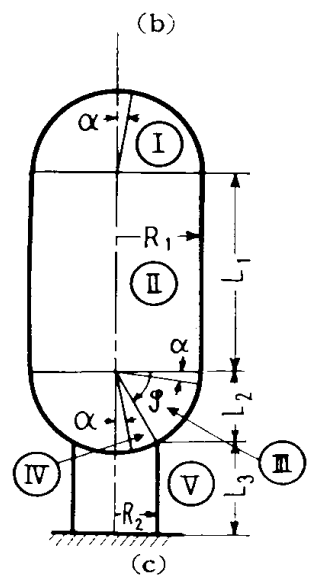

Fig. 17 Models of nuclear vessels 
12 shows the $\mathrm{DET} \sim \Omega$ curves for this case.

(c) Truncated conical shell with free edges

The notations in Fig. 13 are used. The mean radius $R$ is chosen as the reference length $a$, then

$$
\begin{aligned}
& a=R=\left(R_{1}+R_{2}\right) / 2, \Delta=L / R(N-1), \xi=s / R \\
& \rho=(1 / R)\left\{R_{1}+\left(R_{2}-R_{1}\right)(j-1) /(N-1)\right\} \\
& \rho^{\prime}=\left(R_{2}-R_{1}\right) / L, \gamma=\left(R_{2}-R_{1}\right) / \rho L \\
& \omega_{\theta}=\cos (\varphi / 2) / \rho, \omega_{\xi}=0
\end{aligned}
$$

Calculations are carried out for the case of $R=100 \mathrm{~mm}, L=200 \mathrm{~mm}, h=1.0 \mathrm{~mm}, \varphi=0^{\circ}, 40^{\circ}, 80^{\circ}$, $120^{\circ}, 160^{\circ}, 180^{\circ}$. The results for $n=2, N=151$ are shown in Table 3 and Fig. 14. The experimental data in Table 3 are those obtained by Rrof. A. Okumura of Waseda University.

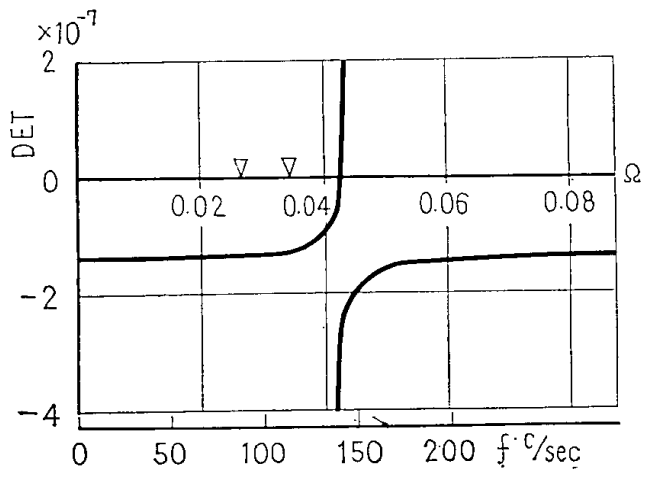

(a)

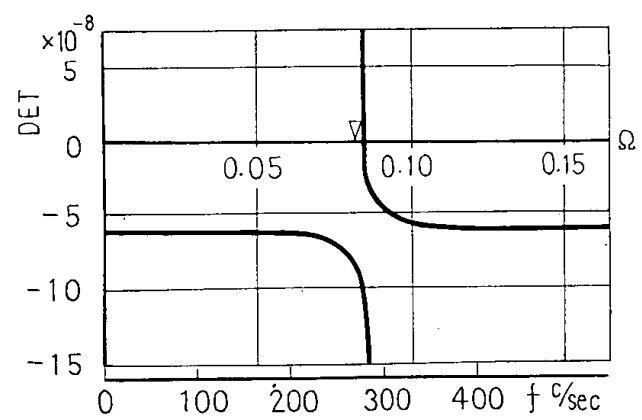

(b)

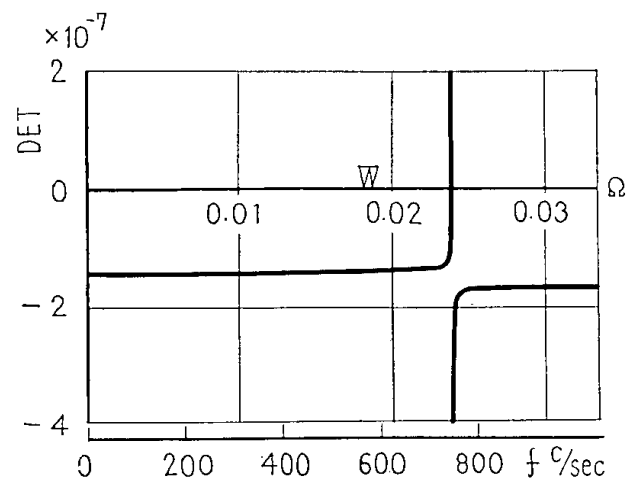

(c)

Fig. 18 DET $\Omega$ curves for shell in Fig. $17(n=1)$ $(\boldsymbol{F}$ : experimental data) (d) Partial spherical shell with fixed edge

The notations in Fig. 15 are used. The radius $R$ is chosen as the reference length $a$, then

$$
\begin{aligned}
& a=R, \Delta=\varphi /(N-1), \xi=\alpha, \\
& \rho=\sin \alpha, \rho^{\prime}=\cos \alpha, \\
& \gamma=\cot \alpha, \omega_{\theta}=\omega_{\xi}=1
\end{aligned}
$$

Calculations are carried out for the case of $R=100 \mathrm{~mm}, h=2.5 \mathrm{~mm}, \varphi=30^{\circ}$. The results for $n=2, N=101$ are shown in Table 4. Figure 16 shows the $\mathrm{DET} \sim \Omega$ curves for this case. The results obtained by $A$. Kalnins ${ }^{(6)}$ are shown in Table 4 and Fig. 16 for comparison.

(e ) Model of nuclear vessel

Three models of nuclear vessels as shown in Fig. 17 are considered. They are made of

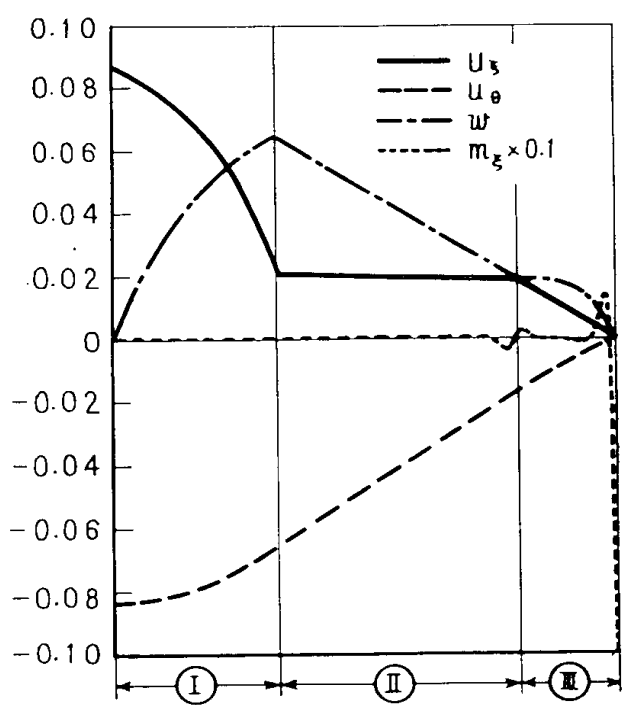

Fig. 19 Distributions of dependent variables for shell in Fig. 17 (a) $(n=1, c=0)$

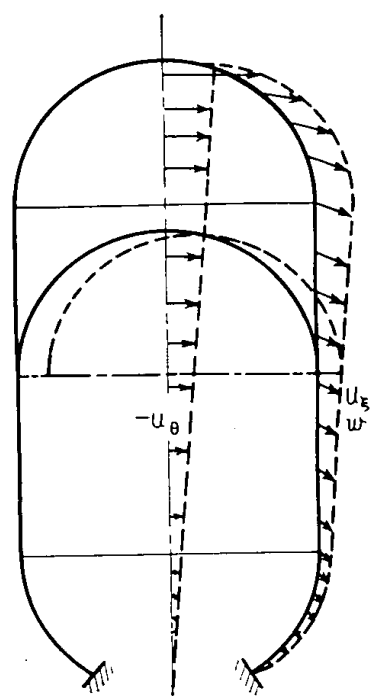

Fig. 20 Mode of free vibration of shell in Fig. 17 (a) $(n=1, c=0)$ 


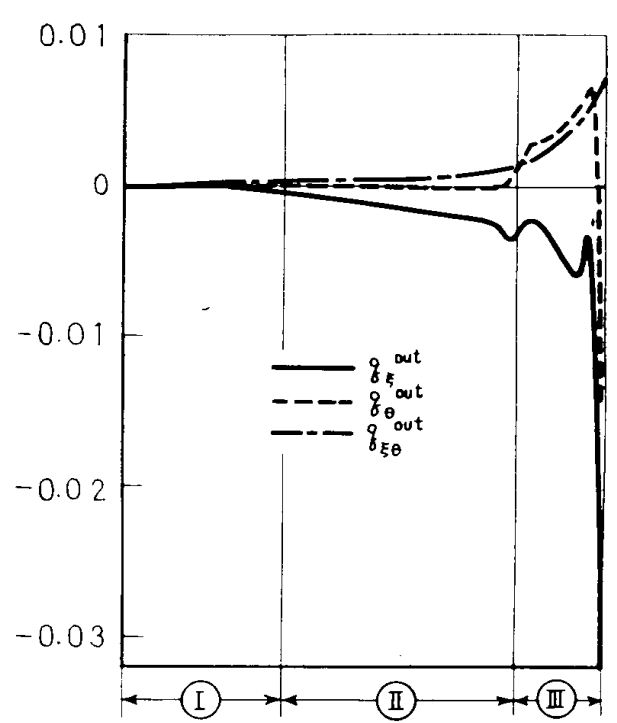

Fig. 21 Distributions of outer stresses of shell in Fig. 17 (a) $(n=1, c=0)$

steel plate of $1.0 \mathrm{~mm}$ thickness, and their height is about $1 \mathrm{~m}$. The $\mathrm{DET} \sim \Omega$ curves of these models for $n=1$ are shown in Fig. 18, where the experimental data by Prof. H. Shibata of Tokyo University are compared with the results by this method. Figure 19 indicates the distributions of $u_{\xi}, u_{\theta}, w$ and $m_{\xi}$ for the model of Fig. 17(a); and Fig. 20 shows the mode of free vibration obtained from the results of Fig. 19 , and Fig. 21 indicates the distributions of stresses on the outer surface of the shell in the above case.

The effect of $N$ on the frequency $\Omega$ is checked in calculations, and it is confirmed that about $200 \sim 400 \mathrm{~N}$ 's give sufficient results.

For free vibrations of cantilever type $(n=1$, $c=0$ ), the numerical results of the natural frequencies are always larger than the experimental data. It is considered that the main reason for this fact is the imperfectness of fixed conditions of the shell. At the fixed edge of the shell, remarkable stress concentrations are found.

Calculations are carried out for cases of $n=2$, i.e. free vibrations in oval forms. In these cases, some disturbances of the distributions of stresses are found in the neighbourhood of the discontinuity points.

\section{Considerations and conclusions}

The numerical method presented by $B$. Budiansky and P.P. Radkowski ${ }^{(1)}$ for problems of unsymmetrical bending of axisymmetric shells is modified to be applicable for problems of free vibrations in this paper, and it is proved by solving numerical examples that the method proposed in this paper is sufficiently applicable for the axisymmetric shells of various kinds.

The effects of the number of the difference points $N$ are examined in the numerical examples. It is found that the time of calculations is approximately proportional to the value of $N$. The suitable value of $N$ in each problem must be decided from one's experiences. In the examples in this paper, it is found that about $100 \sim 300$ $N$ 's give almost appropriate results.

Influences of the number of effective figures in calculations are also examined, and it is found that calculations in ten effective figures give almost satisfactory results.

The weakness of this method is the fact that the forms of the DET $\Omega$ curves are almost flat and bent sharply in the neighbourhoods of the wanted values of $\Omega$. Therefore it is not easy to find out the natural frequencies. To eliminate such weakness of this method, further investigations are necessary hereafter.

In this paper, detailed process for deriving the equations, which are the same as those in ref. ( 1 ), is omitted.

\section{Acknowledgements}

This study is a part of investigations of the Sub-committee on Aseismatic Design on Nuclear Facilities in JSME. sponsored by the Agency of Science and Technology, the chairman of which is Prof. S. Fujii of Tokyo University. The writers are very grateful to the members of the Subcommittee for useful discussions and comments. The computers used in this study are HITAC 5020 in the Calculation Center of Tokyo University, NEAC 2200 in the Calculation Center of Osaka University, and IBM 360 in IBM Osaka Data Center.

\section{References}

(1) Budiansky, B. and Radkowski, P.P., $A I A A J$., Vol. 1 , No. $8(1963-8)$, p. 1833

(2) Sanders, J.L., Jr., NASA, Technical Report, R-24 $(1959-6)$, p. 1 .

(3) Greenbaum, G.A., AIAA J., Vol. 2, No. 3 (1964-3), p. 590 .

(4) Sezawa, K., Theory of Vibration (in Japanese) (1932), p. 121, Iwanamishoten.

(5) Forsberg, K., $A I A A J .$, Vol. 2, No. 12 (1964-12), p. 2150

(6) Kalnins, A., Proc. 4th U.S. Congr. Appl. Mech. (1963), p. 225 . 